\title{
Managing the wildlife tourism commons
}

\author{
Enrico Pirotta ${ }^{1}$ And David Lusseau \\ Institute of Biological and Environmental Sciences, University of Aberdeen, Aberdeen AB24 2TZ United Kingdom
}

\begin{abstract}
The nonlethal effects of wildlife tourism can threaten the conservation status of targeted animal populations. In turn, such resource depletion can compromise the economic viability of the industry. Therefore, wildlife tourism exploits resources that can become common pool and that should be managed accordingly. We used a simulation approach to test whether different management regimes (tax, tax and subsidy, cap, cap and trade) could provide socioecologically sustainable solutions. Such schemes are sensitive to errors in estimated management targets. We determined the sensitivity of each scenario to various realistic uncertainties in management implementation and in our knowledge of the population. Scenarios where time quotas were enforced using a tax and subsidy approach, or they were traded between operators were more likely to be sustainable. Importantly, sustainability could be achieved even when operators were assumed to make simple rational economic decisions. We suggest that a combination of the two regimes might offer a robust solution, especially on a small spatial scale and under the control of a self-organized, operator-level institution. Our simulation platform could be parameterized to mimic local conditions and provide a test bed for experimenting different governance solutions in specific case studies.
\end{abstract}

Key words: common pool resource; management error; management regimes; overexploitation; socioecological simulations; wildlife tourism.

\section{INTRODUCTION}

Natural resources can often be treated as commonpool resources, in that they are subtractable (i.e., one's usage reduces the amount available to others) and nonexcludable (i.e., accessible to everyone; Ostrom 1990). When unmanaged, these resources are likely to become depleted from overuse, i.e., the pursuit of one's shortterm self-interest leads to long-term damages for all. This is traditionally known as the tragedy of the commons (Hardin 1968). An appropriate management of the access and use of such goods can prevent the onset of the tragedy (Ostrom 1990), but each management solution has its own drawbacks and sensitivities (Ostrom et al. 1999).

Wildlife tourism (see Plate 1) has been proposed as an effective way to sustainably exploit wildlife, while contemporaneously improving general public's awareness and attitude toward the natural world (Duffus and Dearden 1990, Tisdell and Wilson 2002). The nonconsumptive use of animals is increasing fast (Reynolds and Braithwaite 2001), especially at sea (e.g., O'Connor et al. 2009), and is generally assumed to positively contribute to addressing conservation issues (Higginbottom and Tribe 2004). However, unregulated tourism can impact animal populations (Reynolds and Braithwaite 2001). Animals perceive tourism as a risk, hence responding to such interactions with anti-preda-

Manuscript received 23 May 2014; revised 22 August 2014; accepted 2 October 2014. Corresponding Editor: E. Nelson

${ }^{1}$ E-mail: enrico.pirotta@abdn.ac.uk tory tactics (Frid and Dill 2002). This can translate into disruptions of their activity budget, spatial displacement, and other sublethal effects that can lead to longterm population consequences (Pirotta et al. 2014). While originally a public good (accessible to everyone [Moore and Rodger 2010], but not subtractable), wildlife targeted by tourism can therefore become a common-pool resource. Tour operators, who aim at maximizing their present benefit, make such resource subtractable by exploiting it to a level at which detrimental effects are instigated, compromising the viability of the wildlife population and hence their future payoffs (Briassoulis 2002, Moore and Rodger 2010). Previous work has identified the required conditions for a successful management of tourism activities, and suggested that tourism does not necessarily lead to the tragedy (Moore and Rodger 2010). However, we currently do not have a framework to understand how to navigate real world situations toward these sustainable conditions.

Wildlife tourism differs from other resource exploitation industries, in that the operators' income is received before the resource is used. Profit can be maximized only through the reduction of costs and the investment in visitors' satisfaction, which can (but not necessarily will) increase the chances of future income (Higginbottom 2004). Moreover, the effects of overexploitation on resource availability generally operate on a longer timescale than the day-to-day trips offered by tour operators (Cumming et al. 2006), so that this resource stops being a public good and 
becomes a common-pool resource only when the viability of the corresponding population starts degrading. In addition, differently from other systems where the industry directly subtracts resource units, the impacts of wildlife tourism occur at the level of the relation between resource units and the overall system. By overconsuming the time spent with the animals, tourism can affect the natural behavior of the animals and therefore compromise their survival or reproductive rates, which could ultimately damage population dynamics (New et al. 2014) and reduce the availability of animals for encounters during tours. This, together with the fact that animals move and need to be kept free, also means that ownership of the resource units is not justified as in other systems and tour operators could solely own the time to spend with the animals, which can only be indirectly depleted.

Because of this complexity, it is difficult to predict operators' behavior as the result of the optimization of simple rules. A simulation approach offers the possibility to investigate the efficacy of different governance solutions, while realistically reproducing the stochasticity intrinsic in such industry. Through simulations, the gradient of outcomes of each management scenario can be explored to pinpoint the variables (and their interactions) that most contribute to its success or failure (Agrawal 2003). Decision makers rarely have all needed facts to make accurate decisions. This uncertainty can affect the outcome of management actions, yet we do not have an evaluation of this effect (Dietz et al. 2003). Conversely, identifying generalities and uncertainties is often difficult using empirical case studies because of the complex interaction between confounding factors. Simulations can also be used to anticipate low probability but high risk outcomes (Dietz et al. 2003).

In this study, we use a simulation approach to evaluate the relative performance of different sets of boundary rules (or policy instruments) in managing wildlife tourism sustainability. We develop a dynamical socioecological simulation framework to assess the effects of these management regimes on a wildlife population targeted by nonconsumptive tourism. Our framework simplifies both the economic model for tourism and the exploitation model for the natural resource, because we aimed at describing the poorly understood interaction between these two components. We investigate under which socioecological conditions each management regime leads to the wildlife population being sustained and the industry remaining viable.

\section{Methods}

\section{Wildlife population}

We assumed that a wildlife population occupied a 50 $\times 50 \mathrm{~km}$ grid of 2500 cells. To simulate a random distribution of animals in space, we generated a spatially correlated random field with an exponential variogram and subsequent kriging (library gstat in R 13.01 [R Development Core Team 2013]; Appendix B: Fig. B1). We varied environmental complexity using the sill and range parameters of the variogram, and used the inverse logit of the random field to define the probability of wildlife encounter in each cell $i\left(p_{i}\right)$. The mean of the kriged surface (hereafter kriged mean) determined the mean probability of encountering the animals across the grid, i.e., kriged mean $=\operatorname{logit}\left(\operatorname{mean}\left(p_{i}\right)\right)$. We then used a binary draw in each cell using $p_{i}$ to generate daily animal distributions.

\section{Tour operators}

Operators started their trips from randomly allocated boundary cells, which were fixed for all trips. We assumed a total of 10 tourist operators working in the area. Each operator offered one trip per day, lasting 1.5 h, charging $£ 15 /$ person. Each trip could accommodate a maximum of 20 tourists. Daily trips were simulated as biased random walks updated every 3 minutes (Fig. 1a). During the first 60 minutes, the trip was directed toward the center of the grid, although this attraction progressively decreased with time. The coordinates of an operator's position at time step $t$ were

$$
X_{t}=X_{t-1}+\operatorname{step} \times \cos (\vartheta) \text { and } Y_{t}=Y_{t-1}+\operatorname{step} \times \sin (\vartheta)
$$

where $\vartheta \sim$ wrapped Cauchy $\left(\mu_{t}, \rho_{t}\right)$. The wrapped Cauchy is a circular distribution often used for turning angles in biased random walks (e.g., Morales et al. 2004). The parameter $\mu_{t}$ was the angle between the $x$ axis and the line joining the current position and the center of the grid and $\rho_{t}$ regulated path directedness (it was inversely related to time, $\rho_{t}=$ time $^{-0.3}$; this simulated an exponential decrease in attraction toward the center of the grid with time ( 0.3 was chosen based on observed realizations of the random walks). Step length was fixed to $1.4 \mathrm{~km}$ per 3 -minute time step (i.e., $28 \mathrm{~km} / \mathrm{h}$ ). After 60 minutes, the process changed, and the biased random walk was directed toward the starting point. Directedness exponentially increased as time passed. When the 90 minutes elapsed, the operator returned to its starting location.

During a trip, an encounter occurred whenever an operator found itself in a cell where, on that day, some animals were present. The proportion of the trip spent with animals influenced tourists' satisfaction (Tisdell and Wilson 2002 and Fig. 1b). Operators decided on encounter duration depending on the number of encounters in the previous day and the desired total time with animals on each day, which was driven by customer satisfaction (tuned here so that 45 minutes corresponded to 0.95 tourists' satisfaction). Encounter duration was drawn from a Weibull distribution with shape $=10$ and scale $=45 / n_{d-1}$, where $n_{d-1}$ was the number of encounters in the previous day. We also simulated animals having more control on their 

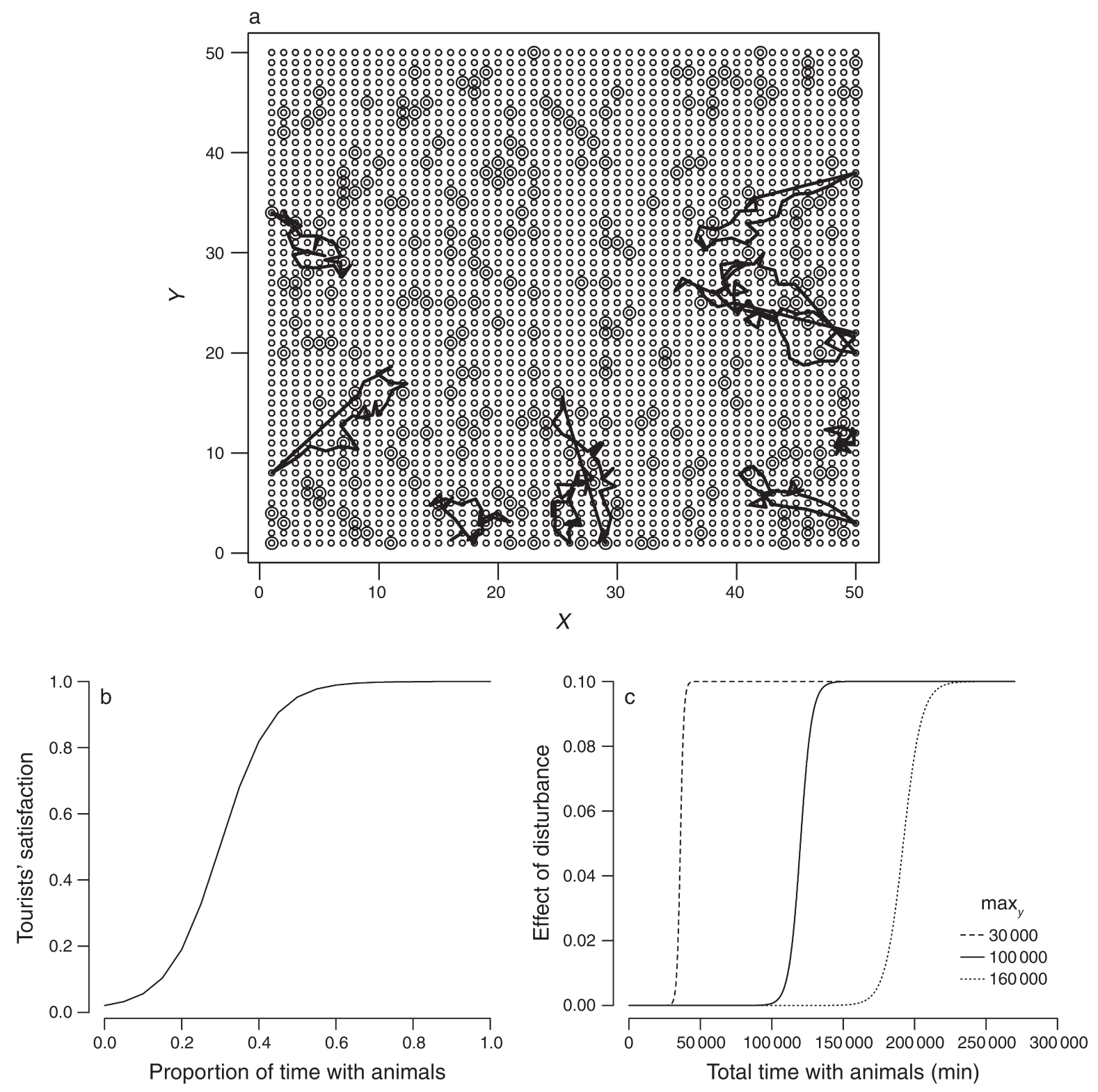

FIG. 1. Visualization of simulation settings. (a) Example of a daily run of the simulation on the spatial grid. The parameters used to define the surface of wildlife distribution were sill $=0.01$ and range $=0.1$. The small dots are the grid cell centers, the lines are the tracks of the operators' trips and the circles represent animal locations on that day. (b) Sigmoid relationship between the proportion of time spent with the animals during a trip and tourists' satisfaction (a unitless measure ranging between 0 [completely unsatisfied] and 1 [completely satisfied]). (c) Sigmoid relationship between total animal exposure to tourism in a year and effect on the mean probability of encounter across the grid (as expressed in Eq. 4). Each line corresponds to a different value of max, i.e., the total amount of time above which there starts to be a relevant effect on the population.

interactions with tourism, i.e., encounter duration was drawn from a Weibull distribution with fixed scale $=$ 20 .

\section{Tourists and profit}

The number of visitors per operator on each day depended on customer satisfaction in the previous day (i.e., a binomial draw using the maximum number of visitors per trip and the value of satisfaction between 0 and 1). If there were no visitors, the operator did not run the trip. We used a simple microeconomic model in which annual profits depended solely on income and running costs

$$
\text { profit }_{o, y}=\text { visitors }_{o, y} \times \text { ticket }^{- \text {timeout }_{o, y}} \times \text { fuel }
$$

where visitors $s_{o, y}$ was the number of visitors in year $y$ per operator $o$, ticket was ticket price, timeout ${ }_{o, y}$ was the sum of the duration of the trips performed by operator $o$ in year $y$, and fuel was fuel cost per unit of time $(0.7 £ / \mathrm{min})$. 


\section{Wildlife use}

The annual growth rate of the wildlife population was $1 \%$, but this was affected by tourism exposure

$$
\begin{aligned}
& \text { effect }_{y}=0.1-0.1 /\left(1+\exp \left(\text { slope }_{y} \times\left(\sum_{o} \text { withanimals }_{o, y}\right.\right.\right. \\
& \left.\left.\left.-\left(\max _{y}+\max _{y} / 5\right)\right)\right)\right)
\end{aligned}
$$

where withanimals ${ }_{o, y}$ was the amount of time spent with the animals by operator $o$ in year $y, \max _{y}$ represented the total amount of time spent with animals above which there started to be a relevant effect on the population,

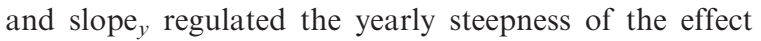
(Fig. 1c). The scaling term $\max _{y} / 5$ was included to ensure that the effect only started after the amount of time spent with animals was greater than $\max _{y}$. Tourism exposure affected the area homogeneously. Therefore, the probability of encountering wildlife in cell $i, p_{i, y}$, changed every year $y$ depending on tourism exposure in the previous year

$$
p_{i, y}=p_{i, y-1} \times\left(\lambda-\text { effect }_{y-1}\right)
$$

where $\lambda$ reflected the annual growth rate of the population (1.01).

As population density changed, the number of animals supporting tourism also varied. The effect of tourism on the population trajectory is mediated by individual cumulative exposure to tourism (Pirotta et al. 2014). Therefore, we varied $\max _{y}$ and slope $y$ with the effective population growth rate to account for changes in per capita exposure resulting from changes in density

$$
\max _{y}=\max _{y-1} \times\left(\lambda-\text { effect }_{y-1}\right) \text { and slope } y=25 / \max _{y} .
$$

Slope ${ }_{y}$ depended on $\max _{y}$ since we expected bigger populations to show a slower response to added disturbance. The relationship between the two parameters was calibrated by visually inspecting the resulting curve.

We assumed the absence of any density-dependent effects on population dynamics. However, the system did have a carrying capacity $\left(\sum_{i} \mathrm{p}_{i, y}=2500\right)$.

\section{Exploitation strategy}

Given an even allocation of exposure, an operator had $\max _{y} / 10$ amount of time to spend with the animals before it started contributing to the overexploitation of this resource. An operator would set a daily limit of time with animals $\left(\max _{y} / 10 / 365\right)$, above which any subsequent encounter on that day had duration equal to 0 .

\section{Defection and cooperation}

A cooperator was defined as an operator that respected its yearly time allowance, while a defector spent more time with animals than it was entitled to. Cooperators and defectors were randomly identified by drawing a fixed proportion of defecting operators at the start of the simulation $(0.1,0.2$, or 0.5$)$. The behavior of operators could then evolve across years in two different ways.

Fixed proportion.-The proportion of defectors did not change within a given year. At the end of year $y$, the operators were more likely to adopt the most successful behavior for year $y+1$, depending on mean profit of defectors and cooperators. Cooperation prevailed deterministically when cooperators' profit was higher, but we also investigated what happened when cooperation arose from a stochastic draw.

Tit-for-tat.-The operators daily updated their behavior on the basis of the behavior of others. The behavior in year $y+1$ arose from a binary draw with probability equal to the proportion of defectors in year $y$.

In both cases, operators needed to know the behavior of others, but an operator's knowledge is not necessarily complete. Hence, we contrasted outcomes depending on whether operators only knew their neighbors' behavior, or they could be informed about the behavior of all operators. In a global knowledge scenario, the profit of defectors and cooperators was evaluated on the entire grid, in a local knowledge scenario only the operators within a radius of $15 \mathrm{~km}$ from an operator's starting location influenced its behavior.

\section{Management regimes}

We simulated five different management regimes defined by a set of boundary rules (or policy instruments) that regulated access to wildlife (Sterner 2003).

1. Open-access.-We tested what happened to the industry and the wildlife population when there was no enforcement of time quotas. Operators were aware of the amount of time they could spend with the animals before contributing to overexploitation, and could decide whether to respect that quota or defect at no cost. This assumed that the maximum time available with animals before any detrimental effect was known without error. To test the sensitivity of the outcome to the precision of this quota, we simulated a situation where this time was estimated with increasing error (1-70\% error). In the real world, operators are likely to sell a consistently unprofitable business. Therefore, we also simulated a situation in which, if an operator's income was negative and had been negative for the previous three years, it would retire from the industry and its quota would be distributed between the remaining operators.

2. Tax (or fine).-This scenario simulated the existence of a managing institution that enforced the time quotas by imposing a fine to defecting operators at the end of each year. The fine reduced a defector's yearly profit, and hence discouraged defection. The fine was proportional to the amount of extra time that each operator spent with animals above its allocated quota. This time is unlikely to be known without error by the institution. We therefore simulated the effect of an increasing error of the institution's evaluation of an operator's extra time by drawing a value in minutes from a Weibull distribution with shape $=1$ and scale $=$ 
1000, 2000, or 5000 (small, medium, and large error), which was subtracted from the operator's extra time. We also tested the effect of a delay in the assessment of the population size and the corresponding safe exposure levels. We assumed that a density survey was carried out every 5,10 , or 20 years and management only updated on those years.

3. Tax and subsidy.-In this scenario, the operators contributed $£ 10000 /$ year to pay for a third party to enforce regulation upon them. The revenue from the annual fines to defectors was then distributed between cooperating operators. We tested the influence of errors in the enforcer's evaluation of operators' behavior, in the same way as we did for the tax regime.

4. Cap.-The operators were not allocated individual quotas, but the managing institution tallied up the overall time spent by all operators with animals. When $\max y$ time was reached, the institution stopped any activity on that year, and no one was allowed to carry out any more trips. In addition, we simulated the error of operators in reporting the time spent with animals on each day. Such error (in minutes) was drawn from a Weibull distribution with shape $=1$ and scale $=1,5,10$ (small, medium, and large error).

5. Cap and trade (individual transferrable quotas).In this scenario, the operators were allocated individual quotas of $\max _{y}$ that they could trade with others for a monetary compensation $(15 £ / \mathrm{min})$. At the start of the simulation, each operator had the same quota. When an operator's cumulative profit was $£ 50000$ in excess to its profit in year 1, that operator was willing to buy extra time shares. Conversely, if an operator's profit on a given year was less than $£ 10000$, the operator wanted to sell part of its shares. In an intermediate situation, an operator did not buy, but could sell if somebody was interested in buying (half of the times, on average). When the remaining quota owned by an operator was less than 1000 minutes per year, the operator liquidated its shares and retired from the business. Unaccounted processes affecting trade (e.g., willingness to sell part of the quota but not all) were simulated by introducing some stochasticity in the amount of quota that was bought and sold. Importantly, trade was assumed to happen before an operator decided on whether to defect or cooperate. The opposite scenario was also investigated.

Each scenario was run for 100 years and for different combinations of other conditions (Appendix A: Table A1). The dynamics of the population and the industry, the trend in customer satisfaction, and the behavior of operators were monitored at each year. We ran scenarios that appeared to be sensitive to stochasticity 10 times, to capture these stochastic effects.

\section{Environment}

We investigated the effects of the environment on the outcome of our simulations by re-running the open access scenario under 27 different combinations of sill, range, and kriged mean parameters. Each parameter could take one of three possible values $(0.01,0.1$, and 1 for sill; $0.1,5,50$ for range; logit(0.01), logit(0.1), logit(0.3) for kriged mean). Relevant parameter combinations were then used to assess the performance of different management regimes under heterogeneous environmental conditions.

\section{RESUlts \\ Open access}

Unsurprisingly, defection rapidly spread between the operators in an open-access scenario, since it led to greater annual profits (Fig. 2a). This resulted in the resource being overexploited, i.e., the targeted wildlife population being extirpated in less than 50 years (Fig. 4a). Consequently, tourism was no longer viable in the area (Fig. 3a). When the operators were allowed to retire from the industry, they did so in less than 30 years. Left undisturbed, the population could then recover from its depletion state (Appendix C: Fig. C1).

When operators followed a tit-for-tat approach, defection could stochastically disappear, which guaranteed a sustainable and profitable industry, but this became progressively less likely as the initial proportion of defectors increased. When there were no defectors, but the maximum allowed exposure before causing an impact was estimated with error, the system showed some resilience. Specifically, an overestimation of the maximum exposure by $30 \%$ still allowed the industry and the population to thrive, but they both collapsed with a $40 \%$ error.

If the animals had some control on the duration of each encounter (i.e., the Weibull distribution for encounter duration had fixed scale $=20$; see Methods: Tour operators), the spread of defection between the operators did not lead to a complete collapse. The population declined and stabilized, which caused the operators' annual profit to also stabilize at lower values. The population and the industry oscillated around a stable state and the operators' annual profits were always positive.

In a local knowledge scenario, cooperation and defection stabilized at a fixed proportion, which varied stochastically depending on the spatial arrangement of the operators and their relative distance. This scenario could cope with some defectors (final proportion $\leq 0.4$; Appendix D: Fig. D1). The initial proportion of defectors had some influence on the final proportion on which the system stabilized, in that lower initial proportions were more likely to stabilize into lower final proportions.

The average probability of encountering the animals across the grid affected the outcome of the simulations. When animals were sparse, e.g., kriged mean $=$ logit(0.01), the operators could not encounter them often enough to sustain a profitable industry. This protected the population from overexploitation and, while annual profits progressively increased, they never turned positive in the 100 simulated years. The wildlife population's spatial assortment had comparably less influence on the 
a) Open access

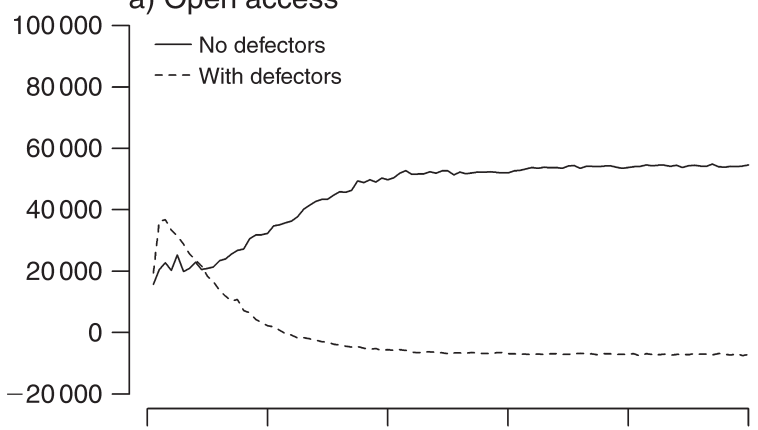

c) Tax and subsidy

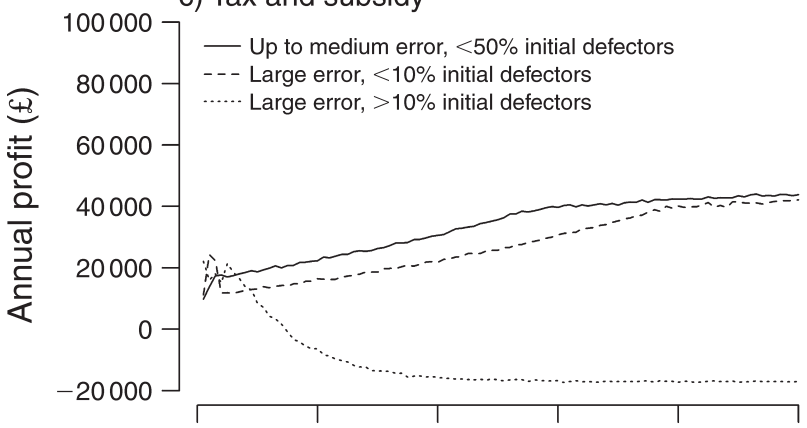

e) Cap and trade

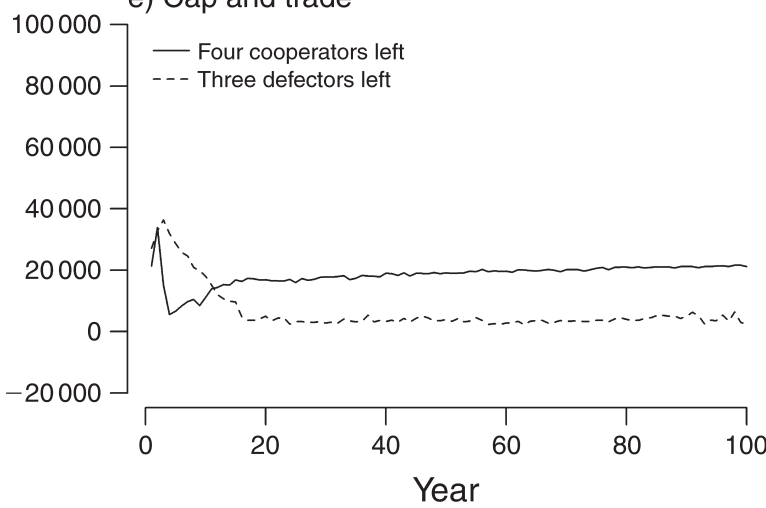

b) Tax

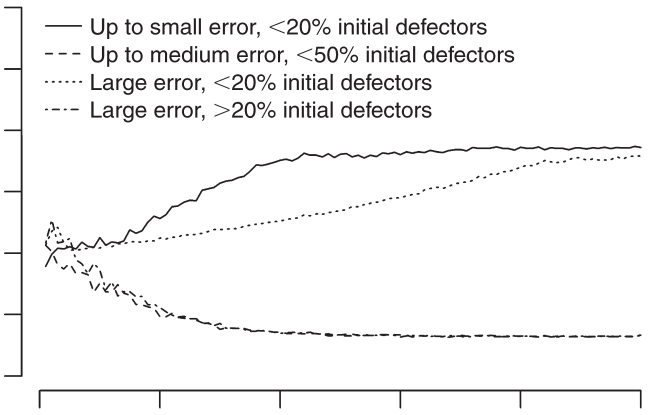

d) Cap

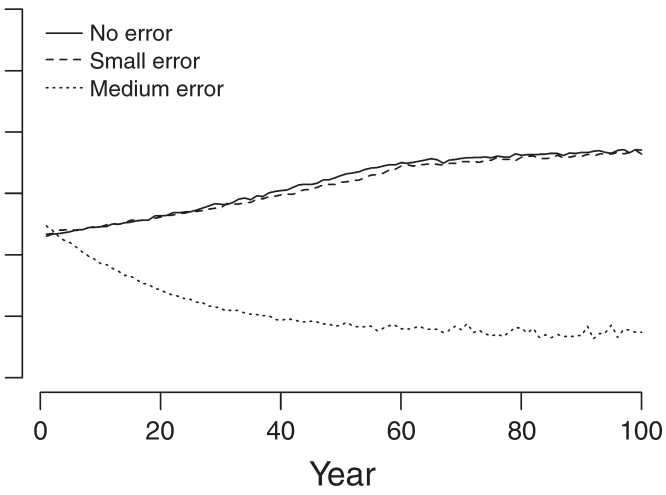

FIG. 2. Simulated temporal trend in operators' annual profit under a selection of different management scenarios: (a) open access, (b) tax, (c) tax and subsidy, (d) cap, (e) cap-and-trade, and combinations of parameters (indicated by the different line types within each plot).

system's dynamics, except for situations in which there was marked spatial heterogeneity in the distribution of the resource $($ range $=5$; sill $=1$ ). This led to differences in profit between operators depending on where they were located on the grid (Appendix E: Fig. E1). We used these conditions to test the effect of environmental heterogeneity on other management regimes.

\section{$\operatorname{Tax}$}

In a scenario where the industry was managed by an institution imposing a fine on defection without any error, defection (starting at any initial proportion) was rapidly overcome by cooperation because of the fines, which made this behavior unprofitable (Figs. 2b, 3b). When coopera- tion arose more stochastically following binary draws, it did not always prevail, since the implemented fine was not large enough to act as a detractor. When there were errors in management but these were small (Weibull scale parameter $=1000$ ) and the initial proportion of defectors was low ( 0.1 or 0.2$)$, cooperation prevailed, although after a longer period. For larger errors or higher initial proportions, defection overcame cooperation, and both the industry and the population collapsed (Figs. 2b, 3b, 4b). Interestingly, for large errors (Weibull scale parameter $=5000)$ and low initial proportions $(0.1$ or 0.2$)$, after an initial population decline, defection was overcome by cooperation, and the system eventually recovered (Figs. $2 b, 4 b)$. This happened because the initial decline was so 
a) Open access

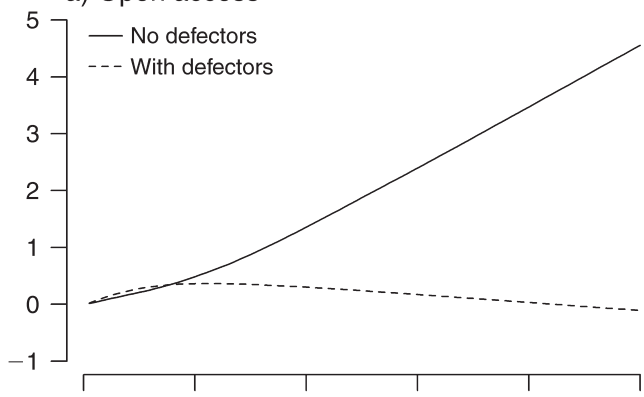

c) Tax and subsidy

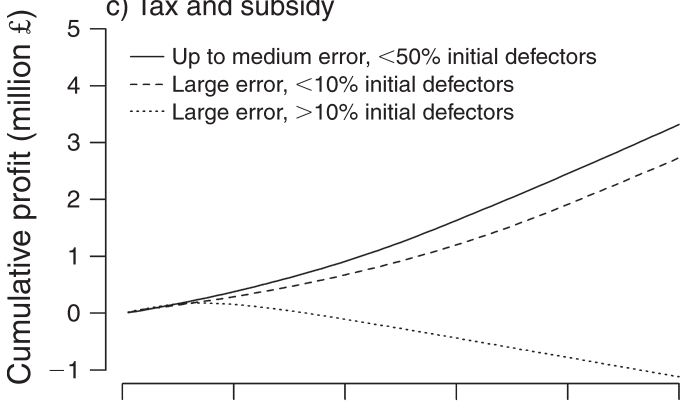

e) Cap and trade

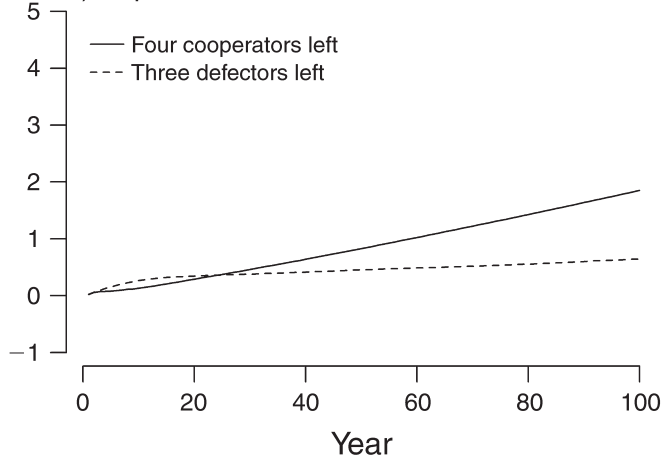

b) Tax

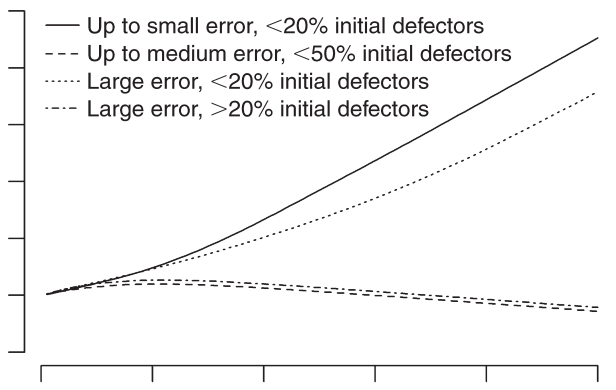

d) Cap

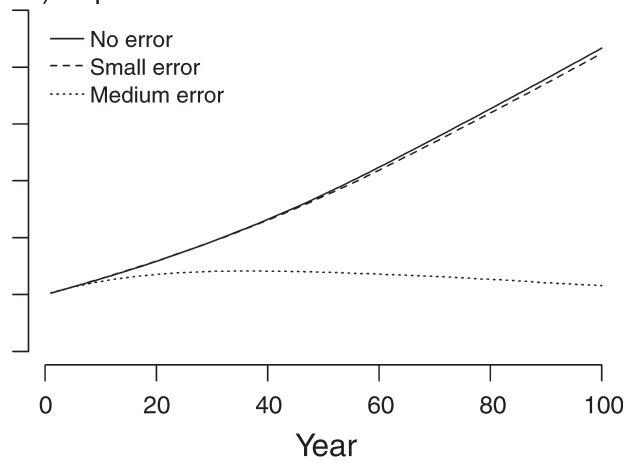

FIG. 3. Simulated temporal trend in operators' cumulative profit under a selection of different management scenarios: (a) open access, (b) tax, (c) tax and subsidy, (d) cap, (e) cap-and-trade, and combinations of parameters (indicated by the different line types within each plot).

dramatic that defection became evident to the institution even with high management error. Because the initial proportion was low, defection did not have time to spread. The outcome of this management scenario remained substantially unchanged when the environment was heterogeneous, although there were differences in overall performance between operators. A delay in the assessment of the safe levels of exposure to tourism also did not change the results. In the absence of management errors, since the population was increasing, such delay only translated into more conservative limits. When there was an error in enforcement, the population declined irrespectively of any delay in management update.

\section{Tax and subsidy}

When cooperating operators received shares of the fines imposed to defectors, the system was more resilient to management errors. The reward for cooperating increased the discrepancy in profit from the defectors, so that even at medium errors (Weibull scale parameter $=$ 2000) and high initial proportion of defectors (0.5), cooperation prevailed. Large errors (Weibull scale parameter $=5000)$ were still compensated if the initial proportion of defectors was low (0.1; Figs. 2c, 3c, 4c). As for the tax regime, the environment did not change these outcomes, but different operators had markedly different profits depending on their location on the grid.

\section{Cap}

Management of the system with an overall cap above which tourism activities were interrupted was successful when there were no reporting errors by the operators. This management solution did accommodate for small errors, but already for medium errors (around 5 minutes 
a) Open access

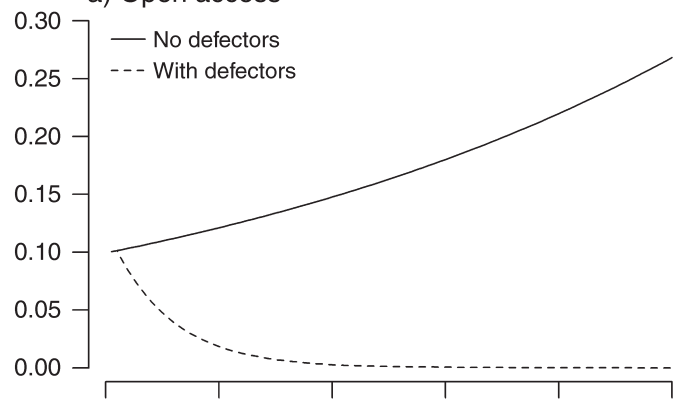

c) Tax and subsidy

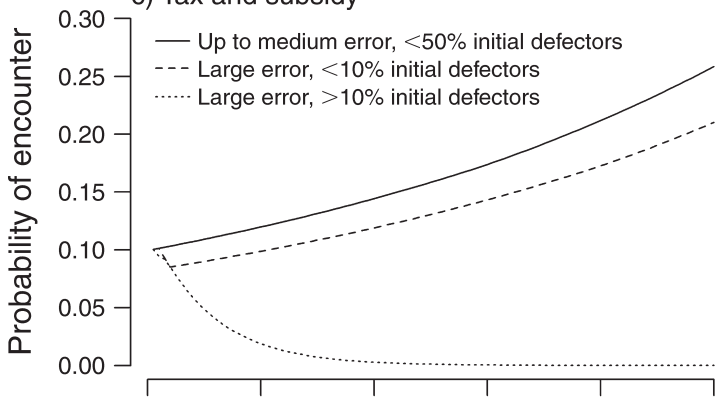

e) Cap and trade

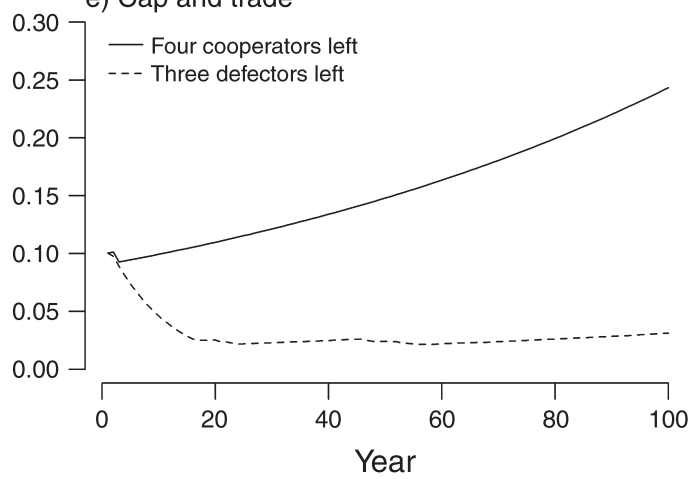

b) Tax

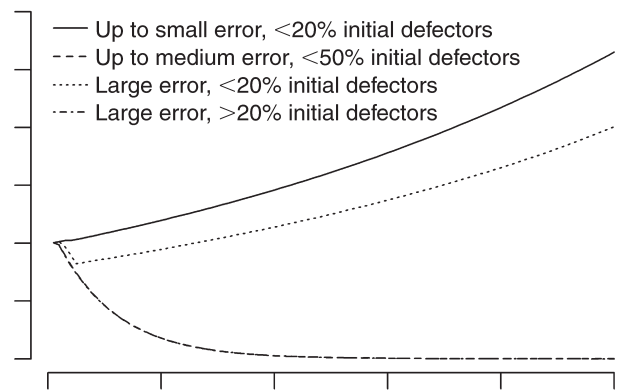

d) Cap

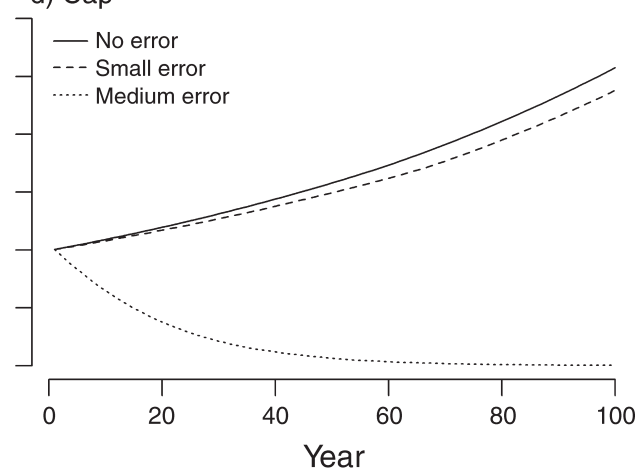

FIG. 4. Simulated temporal trend in mean animal probability of encounter over a selection of different management scenarios (a) open access, (b) tax, (c) tax and subsidy, (d) cap, (e) cap-and-trade and combinations of parameters (indicated by the different line types within each plot).

per operator per day), the industry was shut down with a sufficient delay to cause overexploitation, the animal population's collapse and the subsequent collapse of the industry itself (Figs. 2d, 3d, 4d). The collapse was faster when reporting error was large (Weibull scale parameter $=10$ ). As for the previous scenarios, there was no substantial influence of the environmental conditions.

\section{Cap and trade}

When operators owned their share of the total quota, and the choice of behavior was preceded by a possible trade of these shares between operators, cooperation could spread between the operators and overcome defection (Figs. 2e, 3e, 4e). The number of remaining operators (that had not sold out their entire quota) at the end of the 100-year simulations varied, but individual transferrable quotas only seldom led to a monopoly of one or two operators. This pattern was maintained when operators followed a tit-for-tat approach for their behavior. In this scenario, cooperation spread through a fixed mechanism. First, as a result of overexploitation and consequent decline of the wildlife population, some operators started to lose money and were incentivized to sell their quota. The income they obtained from these sales narrowed the discrepancy between the mean profit of cooperators and defectors, and the choice of defection was thus not straightforward. Cooperators were more affected by wildlife decline, but the sales of their quotas increased their profit, providing an alternative profitable strategy. It follows that if trade happened after an operator had already chosen its behavior for the next year, defection 


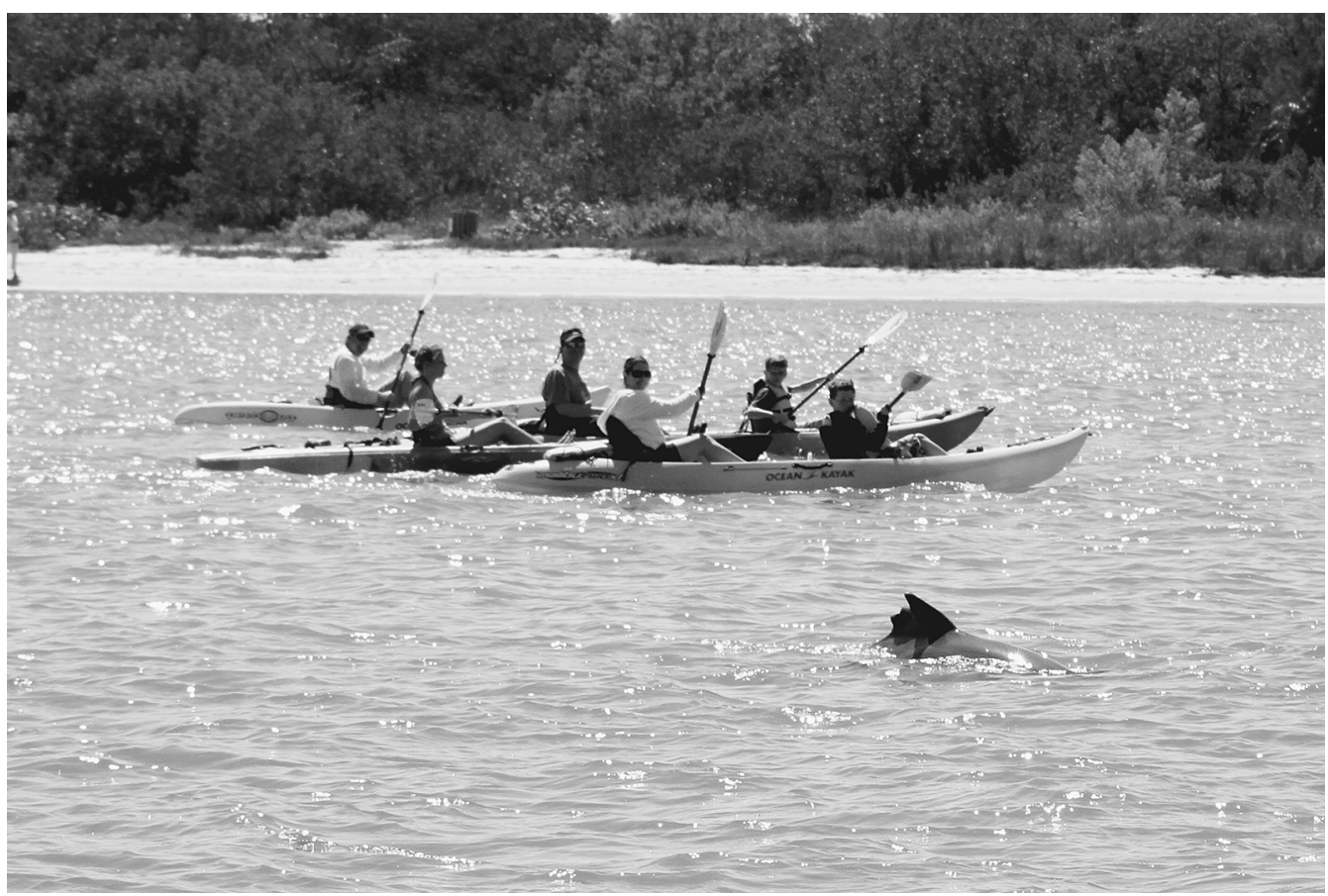

Plate 1. Dolphin watching in the southeastern United States. Photo credit: D. Lusseau.

tended to prevail. The environment did not change the outcome of this scenario.

There was some stochasticity in the outcome of these simulations: the higher the initial proportion of defectors, the lower the chance that this management regime led to the spread of cooperation. Interestingly, even in those cases where defection prevailed on cooperation, the industry never led to a complete collapse of the wildlife population. Because some operators were forced to liquidate their quotas as a result of the resource decline, fewer operators were left in the market. Their impact on the population could not grow indefinitely, even though they did not respect their allocated quotas. Consequently, after an initial decline the population started recovering, unaffected by the few interactions with surviving tourism (Fig. 4e). This is contingent to the assumption that operators did not invest in infrastructure that could, for instance, allow for more and longer trips.

\section{Discussion}

Unregulated wildlife tourism has the potential to cause long-term population consequences on animal populations (Boyle and Samson 1985), leading to both conservation concerns and long-term unprofitability of the industry (Reynolds and Braithwaite 2001). This resource system presents a series of peculiarities, including that subtractability only arises on the longterm, resource units (i.e., the animals) are not directly subtracted but impacts act at a resource-system level, and ownership of resource units is hardly possible.
However, targeted populations that become overexploited by tourism can and should be treated as commonpool resources and managed accordingly (Briassoulis 2002, Moore and Rodger 2010). Using a simulation approach, we tested the outcomes of different management regimes both in terms of the wealth of the industry and the survival of the natural resource. We investigated the sensitivity of such regimes to various realistic uncertainties in management implementation and in our knowledge of the population.

Every regime we have evaluated has the potential to work. Regardless of enforcement mechanisms, if punishments and rewards for defection and cooperation were carefully planned, if we had perfect knowledge of population dynamics and of its resilience to disturbance, and if regulation was enforced without mistakes, the population could survive and grow, and the industry thrive as a consequence. Unfortunately, these conditions are rarely encountered in the real world (Ostrom 1990). First, it is generally hard to assess the amount of disturbance that the population can take before showing some sign of decline. Typically, an incorrect estimation could result from a biased abundance estimate (Buckland et al. 2004) or from a misinformed link between the observable short-term effects of tourism on the animals and the long-term population-level consequences (Gill et al. 2001). We showed here that a perfect management scheme is resilient to an overestimation of such limit by $30 \%$, which, for example, could accommodate the error of an abundance estimate with standard precision (e.g., Taylor et al. 2007). However, management implemen- 
tation is also generally far from perfect, especially when this is carried out by a central institution that the operators perceive as a remote entity (Ostrom 1990). Our simulations showed that even small errors in imposing fines (in this case, an underestimation of time above the limit of less than 3 minutes/day on average per operator) could lead to the collapse of the system for a large initial proportion of defectors. A cap management scheme was also only resilient to small errors. Given the levels of unreported or misreported harvest in other industries targeting natural resources (Yablokov 1994, Watson and Pauly 2001, Pitcher et al. 2002, Gavin et al. 2010), aiming at such high monitoring accuracy is unrealistic (Keane et al. 2008). A tax and subsidy approach offered a more robust scheme where operators contributed to enforcement and received a reward if they cooperated. Despite these improvements, large errors in management (which could play a role, for example, in remote, hard to monitor locations) combined with high initial proportion of defecting operators were still leading to overexploitation and industry collapse.

Generally, our results showed that errors in management had a stronger influence on the outcome of a scenario than the initial attitude of the agents, so that scenarios with poor management were expected to fail even if the initial number of defectors was low. The inefficacy and unfairness of the management in place might themselves promote defective behaviors (Ostrom 1990). On the other hand, some simulations led to a positive outcome by chance (i.e., defection stochastically disappearing from the system). In the real world, defective operators could also leave the industry for reasons other than profit. While defection could reappear in a real system, this result emphasizes the need to treat single case studies with caution.

In our simulations, defecting operators would cause the failure of the industry before the complete depletion of the exploited resource. In those scenarios where they could retire, the wildlife population was then able to recover.

Environmental variability can alter the profitability of the industry (Briassoulis 2002). In our simulations, large heterogeneity caused different operators to perform differently. In an extreme case, resource heterogeneity could make portions of the population's range unprofitable from a business perspective. Although this was not implemented in our simulations, such heterogeneity could concentrate the pressure of the industry on smaller areas, possibly causing the displacement of the animals to other, undisturbed areas (Lusseau 2005, Bejder et al. 2006). We also found that the basal encounter rate was, as expected, very important in guaranteeing a sufficient customer satisfaction. Quick and unpredictable changes in the spatial dynamics of the animals, which might happen for example if the population's range is much wider than the area covered by tourism, can therefore lead to sudden drops in customer satisfaction and to a rapid decline of the industry (Briassoulis 2002). Low availability or predictability of a wildlife resource may, in a real world, contribute to support defecting behavior, since operators have lower benefits at stake.

We found that local knowledge (as opposed to complete information) had the potential to stabilize these dynamics. The structure it introduced in the operators' network promoted the retention of cooperation. Even in the absence of any form of management, restricted access to information about the behavior of others could sustain a profitable industry while maintaining a growing wildlife population. This result confirmed previous work showing that any form of structure in the interactions between the different players can lead to cooperation fixation in populations (Ohtsuki et al. 2006).

The privatization of natural resources has been repeatedly proposed as a solution to the inevitable issues associated with the implementation of external regulation (e.g., Smith 1981), although it can raise ethical concerns (Luck et al. 2012). Tradable quotas of a resource that different agents can buy and sell performed successfully in some contexts (Costello et al. 2008). Individual transferable quotas are proposed to increase stewardship incentives because they guarantee long-term financial benefit from harvesting a resource sustainably. While empirical evidence supports this system in some cases, the mechanism by which it operates is unclear. Overexploiting a resource while others respect their owned quota still leads to greater benefits for the defector. Therefore, it is still unclear what might prevent an operator from free-riding (Parslow 2010). Privatizing wild animals is an unrealistic solution, but here we explored how the system would perform if quotas of the total amount of time available with the animals could be traded between operators. Our results suggest that the trade itself might offer a proximate explanation to the success of this regime. In our simulations, tradable quotas promoted cooperation by increasing an operator's payoff in a given year without the need for him to defect. They allowed operators to make money in the short term. This prevented them from defecting since, at a given time, defection was less convenient than selling part of the quota when profit started to decline. In a real world, operators are also likely to prefer a guaranteed income resulting from selling their shares to the risks associated with defection. Crucially, this system holds only when the value of the quota is evaluated fairly, and a sale compensates for future potential profits. Also, the decision to defect must take into account the income derived from the sales, i.e., trade has to happen before an operator choose its behavior for the following year, otherwise it does not provide any perceived benefit and does not favor cooperation. In more complex microeconomic models, the profit surplus generated by the sale could be invested in infrastructure. These capital investments can then protect profit for the future, and therefore act as what others defined as stewardship 
incentives. Nevertheless, it should be noted that, at times, our simulations would stochastically lead to the spread of defection, the exclusion of cooperating operators from the industry, and the confinement of the population into a depleted stable state. This would likely deteriorate to the population's collapse in a realworld scenario, where successful operators would invest on enlarging their business. Also, our model did not treat the fair allocation of initial quotas, which is known to be a critical issue in certain cases (Ostrom et al. 1999).

\section{Successful management schemes and institutions}

Our results showed that no single management scheme could consistently support the spread of cooperative behavior, the development of a profitable industry and the survival of the targeted population. It has been suggested that successful governance should rely on a combination of different management arrangements (Dietz et al. 2003). When using a simple combination of the two most successful regimes (tax and subsidy and cap and trade) as an exploratory exercise, we indeed found that for high management errors and high initial proportion of defectors (i.e., when the tax and subsidy regime tended to fail), the operators remaining in the market maintained a profitable business without depleting the wildlife population. Moreover, such combination stabilized the spread of cooperation, which appeared to stochastically fail when a market regime based on individual transferrable quotas was in use (Appendix F: Fig. F1). Therefore, in these conditions it emerges that each regime is able to take over when the other regime would drive the system in a disadvantageous state, improving management performance. Such combined management is likely to become even more robust if it is implemented on a small scale, where local knowledge could promote the development of strong pockets of cooperators (Appendix D: Fig. D1). In the real world, small-scale management will also be easier to enforce and will increase the role of social capital in determining behavior (Dietz et al. 2003).

We have explored the strengths and weaknesses of various policy instruments. The next step would be to identify the best institutional arrangements that could facilitate the implementation of such management measures. Given the nature of the resource units (i.e., wild, free-ranging animals), it is likely that ownership of such units will remain impossible and undesirable, and any institution should instead regulate the distribution of quotas of time to spend with the animals. The enforcement of taxes and fines by a central institution is likely to suffer from substantial errors (Ostrom 1990), while a form of self government (e.g., a cooperative of tour operators acting locally) could successfully implement a tax and subsidy scenario. This is also expected to be reinforced by the operators' greater sense of ownership and responsibility, and by personal and societal values that come into play when people interact more closely (Ostrom 1990, Ostrom et al. 1999). The privately hired enforcers will also likely be more efficient at monitoring defection than a central public institution (Ostrom 1990), and the users will be incentivized to support rule enforcement (Dietz et al. 2003). This would decrease errors, increasing the propensity for sustainability. In order to stabilize these dynamics, the operators could autonomously institute an agreement where shares of the time quota can be traded, in order to profit from the strengths of a cap-and-trade regime.

\section{Limitations and assumptions}

While our economic model for the tourism industry and our model for the exploitation of the natural resource were both simple, we focused our attention on investigating the complex connection between the two, which has previously received little attention. Our model, like all models, is a simplification of reality and involves some assumptions about the system. For example, we assumed that the effect of disturbance was distributed homogeneously across the grid, while there is evidence that wildlife tourism can have local consequences, which then cause spatial rearrangements of the animals (Bejder et al. 2006). Similarly, while we considered the effects of a spatially heterogeneous distribution of the animals, we did not investigate what would happen if the operators could know where the animals were, retain and improve such knowledge over time, and share it with other operators. In a real-world scenario, animal distribution is likely to be nonhomogeneous, causing a varying profitability of different areas, which might also change in time (Bennett et al. 2009). Therefore, operators' movement patterns would likely become directed toward areas with higher probability of encountering the animals, potentially increasing the impact on the population and altering operators' interactions and income. This and other components of stochasticity we have introduced in our simulation do not reflect actual stochastic elements in the system, but rather our lack of knowledge about its real socioecological dynamics, with unknown implications on the outcome of our simulations. It is likely that these elements will also differ on a case-by-case basis. Future work could reduce such uncertainty by parameterizing these processes using real-world observations and explore how the diversity of processes influences outcomes. Moreover, we excluded natural variability in the population's growth rate, as well as the presence of other human stressors that might act synergistically with tourism and alter the outcome of such interactions (MacDonald 2000). We argue that these are necessary simplifications of the natural component of the system to pinpoint the sensitivities of different management scenarios in the absence of confounding factors. In addition, we did not consider any factor affecting tourists' numbers other than their satisfaction from the trips. For example, cooperating operators may be rewarded with a "green label," which, in our simulation, 
could increase tourists' satisfaction irrespective of the amount of time spent with animals. It is easy to see how such modification would make any management scenario more robust to errors and promote cooperation. On the other hand, a reduction in overall tourism in an area would probably push the operators toward defection, in order to compensate for lower numbers with higher satisfaction. Moreover, in the cap-and-trade scenario, we did not allow the price of the time quotas to vary as the result of supply and demand. Finally, we did not account for the influence of societal, moral, and personal values in operator behavior, and assumed that they made rational decisions based exclusively on their expected profits. Any effect of these additional aspects on the management outcome should be formally analyzed in the future, but will also be highly contextdependent (Agrawal 2001). Moreover, we expect these values to be reinforced by successful management and become less influential under unfair management.

\section{Implications for the development of tourism management scenarios}

We showed that a wildlife population overexploited by tourism should be viewed as a common-pool resource. We can use simulations to assess the potential outcomes of its management to disentangle the complex interactions of several confounding factors that affect the socio-ecological outcomes. Our simulations offer a predictive platform that could be adapted to specific case studies. The model could be parameterized to mimic local conditions and provide a test-bed for experimenting different governance solutions when such experimentation is not possible in the real world. We can conclude that we cannot ignore the sensitivity of the outcome of different management scenarios to various uncertainties associated with the success of the implementation of regulation. These should be realistically taken into account when selecting a specific strategy in a given context, in order to avoid the onset of the tragedy of the commons. Scenarios where time quotas were enforced using a tax and subsidy approach, or were traded between operators appeared to be more robust than other forms of management. We suggest that a mixed strategy where the elements of strength from these regimes are combined (possibly on a small spatial scale and under the control of an association of cooperating operators for their mutual benefits) might offer an effective and sustainable solution (Dietz et al. 2003). Interestingly, the success of these scenarios emerged under simple rational decision assumptions and did not require questioning of the assumption that individuals might be "selfish, norm-free, and maximizers of shortrun results" (Ostrom et al. 1999). However, complex context dependencies are likely to characterize realworld systems and should caution from considering these solutions as panaceas (Ostrom et al. 2007). Empirical observations are now needed to tune our general model to account for these more complex local factors.

\section{ACKNOWLEDGMENTS}

This work received funding from the MASTS pooling initiative (the Marine Alliance for Science and Technology for Scotland) and their support is gratefully acknowledged. MASTS is funded by the Scottish Funding Council (grant reference HR09011) and contributing institutions. This work was stimulated by discussions with the Moray Firth Dolphin Space Programme and we particularly thank Ben Leyshon (Scottish Natural Heritage) for fruitful discussions. The authors would like to thank K. Barton and C. Konrad for their advice on biased random walks and correlated random fields, D. Murphy for useful discussions during the development of the simulations, and M. Marcoux for important comments on an earlier version of this work. Finally, we thank two anonymous reviewers, whose comments have greatly improved the manuscript.

\section{Literature Cited}

Agrawal, A. 2001. Common property institutions and sustainable governance of resources. World Development 29:16491672.

Agrawal, A. 2003. Sustainable governance of common-pool resources: context, methods, and politics. Annual Review of Anthropology 32:243-262.

Bejder, L., A. Samuels, H. Whitehead, N. Gales, J. Mann, R. Connor, M. Heithaus, J. Watson-Capps, C. Flaherty, and M. Krützen. 2006. Decline in relative abundance of bottlenose dolphins exposed to long-term disturbance. Conservation Biology 20:1791-1798.

Bennett, V. J., M. Beard, P. A. Zollner, E. Fernández-Juricic, L. Westphal, and C. L. LeBlanc. 2009. Understanding wildlife responses to human disturbance through simulation modelling: A management tool. Ecological Complexity 6:113-134.

Boyle, S. A., and F. B. Samson. 1985. Effects of nonconsumptive recreation on wildlife: a review. Wildlife Society Bulletin 13:110-116.

Briassoulis, H. 2002. Sustainable tourism and the question of the commons. Annals of Tourism Research 29:1065-1085.

Buckland, S. T., D. R. Anderson, K. P. Burnham, J. L. Laake, D. L. Borchers, and L. Thomas. 2004. Advanced distance sampling: estimating abundance of biological populations. Oxford University Press, Oxford, UK.

Costello, C., S. D. Gaines, and J. Lynham. 2008. Can catch shares prevent fisheries collapse? Science 321:1678-1681.

Cumming, G., D. Cumming, and C. Redman. 2006. Scale mismatches in social-ecological systems: causes, consequences, and solutions. Ecology and Society 11(1):14.

Dietz, T., E. Ostrom, and P. C. Stern. 2003. The struggle to govern the commons. Science 302:1907-1912.

Duffus, D., and P. Dearden. 1990. Non-consumptive wildlifeoriented recreation: a conceptual framework. Biological Conservation 53:213-231.

Frid, A., and L. Dill. 2002. Human-caused disturbance stimuli as a form of predation risk. Conservation Ecology 6:11.

Gavin, M. C., J. N. Solomon, and S. G. Blank. 2010. Measuring and monitoring illegal use of natural resources. Conservation Biology 24:89-100.

Gill, J. A., K. Norris, and W. J. Sutherland. 2001. Why behavioural responses may not reflect the population consequences of human disturbance. Biological Conservation 97:265-268.

Hardin, G. 1968. The tragedy of the commons. Science 162:1243-1248.

Higginbottom, K., editor. 2004. Wildlife tourism: impacts, management and planning. Common Ground Publishing, 
Queensland Cooperative Research Centre for Sustainable Tourism, Altona, Victoria, Australia.

Higginbottom, K., and A. Tribe. 2004. Contributions of wildlife tourism to conservation. Pages 99-123 in K. Higginbottom, editor. Wildlife tourism: impacts, management and planning. Common Ground Publishing, Queensland Cooperative Research Centre for Sustainable Tourism, Altona, Victoria, Australia.

Keane, A., J. P. G. Jones, G. Edwards-Jones, and E. J. MilnerGulland. 2008. The sleeping policeman: understanding issues of enforcement and compliance in conservation. Animal Conservation 11:75-82.

Luck, G. W., K. M. A. Chan, U. Eser, E. Gómez-Baggethun, B. Matzdorf, B. Norton, M. B. Potschin, K. A. I. M. A. Chan, and U. T. A. Eser. 2012. Ethical considerations in on-ground applications of the ecosystem services concept. BioScience 62:1020-1029.

Lusseau, D. 2005. The state of the scenic cruise industry in Doubtful Sound in relation to a key natural resource: bottlenose dolphins. Pages 246-262 in C. M. Hall and S. Boyd, editors. Nature-based tourism in peripheral areas: development or disaster? Channelview Publications, London, UK.

MacDonald, L. 2000. Evaluating and managing cumulative effects: process and constraints. Environmental Management 26:299-315.

Moore, S. A., and K. Rodger. 2010. Wildlife tourism as a common pool resource issue: enabling conditions for sustainability governance. Journal of Sustainable Tourism 18:831-844.

Morales, J. M. J., D. D. T. Haydon, J. Frair, K. E. Holsinger, and J. M. Fryxell. 2004. Extracting more out of relocation data: building movement models as mixtures of random walks. Ecology 85:2436-2445.

New, L. F., et al. 2014. Using short-term measures of behaviour to estimate long-term fitness of southern elephant seals. Marine Ecology Progress Series 496:99-108.

O'Connor, S., R. Campbell, H. Cortez, and T. Knowles. 2009. Whale watching worldwide: tourism numbers, expenditures and expanding economic benefits. Prepared by Economists at Large, Melbourne Australia. International Fund for Animal Welfare, Yarmouth, Massachusetts, USA.

Ohtsuki, H., C. Hauert, E. Lieberman, and M. A. Nowak. 2006. A simple rule for the evolution of cooperation on graphs and social networks. Nature 441:502-505.
Ostrom, E. 1990. Governing the commons: the evolution of institutions for collective action. Cambridge University Press, Cambridge, UK.

Ostrom, E., J. Burger, C. B. Field, R. B. Norgaard, and D. Policansky. 1999. Revisiting the commons: local lessons, global challenges. Science 284:278-282.

Ostrom, E., M. A. Janssen, and J. M. Anderies. 2007. Going beyond panaceas. Proceedings of the National Academy of Sciences USA 104:15176-15178.

Parslow, J. 2010. Individual transferable quotas and the "tragedy of the commons." Canadian Journal of Fisheries and Aquatic Sciences 67:1889-1896.

Pirotta, E., L. New, J. Harwood, and D. Lusseau. 2014. Activities, motivations and disturbance: An agent-based model of bottlenose dolphin behavioral dynamics and interactions with tourism in Doubtful Sound, New Zealand. Ecological Modelling 282:44-58.

Pitcher, T. J., R. Watson, R. Forrest, H. P. Valtysson, and S. Guenette. 2002. Estimating illegal and unreported catches from marine ecosystems: a basis for change. Fish and Fisheries 3:317-339.

R Development Core Team. 2013. R: a language and environment for statistical computing. R Foundation for Statistical Computing, Vienna, Austria. http://www. R-project.org/

Reynolds, P. C., and D. Braithwaite. 2001. Towards a conceptual framework for wildlife tourism. Tourism Management 22:31-42.

Smith, R. 1981. Resolving the tragedy of the commons by creating private property rights in wildlife. Cato Journal $1: 439-468$.

Sterner, T. 2003. Policy instruments for environmental and natural resource management. Resources for the Future Press, Washington, D.C., USA.

Taylor, B. L., M. Martinez, T. Gerrodette, J. Barlow, and Y. N. Hrovat. 2007. Lessons from monitoring trends in abundance of marine mammals. Marine Mammal Science 23:157-175.

Tisdell, C., and C. Wilson. 2002. Ecotourism for the survival of sea turtles and other wildlife. Biodiversity and Conservation 11:1521-1538.

Watson, R., and D. Pauly. 2001. Systematic distortions in world fisheries catch trends. Nature 414:534-536.

Yablokov, A. V. 1994. Validity of whaling data. Nature 367:108.

\section{Supplemental Material}

\section{Ecological Archives}

Appendices A-F are available online: http://dx.doi.org/10.1890/14-0986.1.sm 